\title{
Bending response of FGPM plates under voltage load
}

\author{
Ladislav Sátor ${ }^{1, *}$, Vladimír Sládek ${ }^{1}$, and Ján Sládek ${ }^{1}$ \\ ${ }^{1}$ Institute of Construction and Architecture, Slovak Academy of Sciences, 84503 Bratislava, Slovakia
}

\begin{abstract}
In this paper, we investigate the transient response of thin FGPM plates under voltage load within classical theory of the plates. The material properties of plate (such us elastic, piezoelectric, dielectric coefficients and mass density) are functionally graded in the in-plane direction. The strong form meshless formulations for solution of considered initial-boundary value problems are developed in combination with Moving Least Squares (MLS) approximation technique. Several numerical experiments are presented for the investigation of the influence of in-plane gradations of material coefficients on the transient response of the FGPM plates.
\end{abstract}

\section{Introduction}

Nowadays, the smart materials and structures, such as piezoelectric ones are commonly used in several branches of engineering practice like sensors and actuators for monitoring and/or selfmonitoring of the health of structures $[1,2]$. These materials are usually made as laminated composites from ceramic slices. It is well known the discontinuities on interfaces between two layers in such a composites lead to concentration of gradient fields, which is the significant factor of failure of laminated structures. There is a strong motivation to replace laminated piezoelectric plate structures by functionally graded ones, if possible. Therefore the study of behavior of functionally graded piezoelectric (FGPM) plates under voltage loadings has become attractive, what can be confirmed by a huge amount of papers devoted to piezoelectric analyses with using various plate bending theories for FGPM plates under static and/or dynamic loadings [3-7]. In this paper, the bending of thin FGPM plates with in-plane gradation of material coefficients under voltage loading is considered within the classical theory of plates. Note that the in-plane gradation of material coefficients makes the equations of motion much more complicated due to the fact they are given by the partial differential equations (PDE) with variable coefficients. The equations of motion which are given by the 4th order PDE are decomposed into the 2nd order PDEs in order to overcome the inaccuracy of approximation of high order derivatives of field variables. The strong form meshless formulations in combination with Moving Least Squares (MLS) approximation scheme [8] are developed for solution of boundary value problems for FGPM plates. The attention is paid to the numerical investigation of the

\footnotetext{
* Corresponding author: ladislav.sator@savba.sk
} 
influence of in-plane gradations of various material coefficients on the electric potential field, deflection and/or in-plane displacements of the FGPM plate.

\section{Equations of motion for FGPM plates}

Let us consider a transversally isotropic FGPM plate [9] with the power-law in-plane gradation of elastic, piezoelectric, dielectric material coefficients and mass density as:

$$
\begin{gathered}
c_{a b}(\mathbf{x})=c_{a b(0)} c_{(H)}^{E}(\mathbf{x}), \quad c_{(H)}^{E}\left(x_{1}\right)=1+\zeta\left(\frac{x_{1}}{L}\right)^{p} \quad a, b=\{1,2,3,4,5,6\} \\
e_{a b}(\mathbf{x})=e_{a b(0)} e_{(H)}(\mathbf{x}), \quad e_{(H)}\left(x_{1}\right)=1+\xi\left(\frac{x_{1}}{L}\right)^{r} \\
\chi_{i j}^{\varepsilon}(\mathbf{x})=\chi_{i j(0)}^{\varepsilon} \chi_{(H)}^{\varepsilon}(\mathbf{x}), \quad \chi_{(H)}^{\varepsilon}\left(x_{1}\right)=1+\lambda\left(\frac{x_{1}}{L}\right)^{s} \quad i, j=\{1,2,3\} \\
\rho(\mathbf{x})=\rho_{0} \rho_{(H)}(\mathbf{x}), \quad \rho_{(H)}\left(x_{1}\right)=1+\varepsilon\left(\frac{x_{1}}{L}\right)^{g},
\end{gathered}
$$

where $\zeta, \xi, \lambda, \varepsilon$ and $p, r, s, g$ are the values of the level and exponent of the power-law gradation, respectively.

Before writing of the equations of motion in Kirchhoff - Love (classical) theory (KLT) of thin plates for piezoelectric initial-boundary value problems (IBVP) we define the dimensionless coordinates and time variable as

$$
x_{\beta}^{*}:=\frac{x_{\beta}}{L}, \quad x_{3}^{*}:=\frac{x_{3}}{h_{0}}=h^{*}(\mathbf{x}) z, h(\mathbf{x})=h_{0} h^{*}(\mathbf{x}), \quad t^{*}:=\frac{t}{T}
$$

with $L, h_{0}, T$ being the characteristic in-plane dimension, plate thickness and time interval. The dimensionless field variables in the KLT are the in-plane displacements, deflections and electric potential, which are introduced as

$$
\begin{gathered}
u_{\beta}^{*}(\mathbf{x}, t):=\frac{u_{\beta}(\mathbf{x}, t)}{h_{0}}, w^{*}(\mathbf{x}, t):=\frac{w(\mathbf{x}, t)}{h_{0}}, \phi_{0}^{*}(\mathbf{x}, t):=\frac{\phi_{0}(\mathbf{x}, t)}{\Phi_{0}}, \\
\Phi^{+^{*}}(\mathbf{x}, t):=\frac{\Phi^{+}(\mathbf{x}, t)}{\Phi_{0}}, \Phi^{-*}(\mathbf{x}, t):=\frac{\Phi^{-}(\mathbf{x}, t)}{\Phi_{0}}
\end{gathered}
$$

where we have omitted the superscript $*$ in coordinates and time variable and three fields $\phi_{0}^{*}(\mathbf{x}, t), \Phi^{+*}(\mathbf{x}, t), \Phi^{-*}(\mathbf{x}, t)$ occur in the truncated expansion of the electric potential w.r.t. the $z$-coordinate: $\phi^{*}(\mathbf{x}, z, t) \approx\left(1-4 z^{2}\right) \phi_{0}^{*}(\mathbf{x}, t)+z \Phi^{{ }^{-}-}(\mathbf{x}, t)+2 z^{2} \Phi^{{ }^{*}+}(\mathbf{x}, t)$. The expression $\Phi^{ \pm}(\mathbf{x}, t)$ is staying for $\Phi^{ \pm}(\mathbf{x}, t)=\phi^{+}(\mathbf{x}, t) \pm \phi^{-}(\mathbf{x}, t)$, where $\phi^{+}(\mathbf{x}, t)$ and $\phi^{-}(\mathbf{x}, t)$ is the electric potential prescribed on the top and bottom surface of the plate, respectively.

Combination of the variational formulation for piezoelectric problems with assumptions of the KLT for thin plates yields the strong form of the equations of motion in view of the PDE 


$$
\begin{gathered}
T_{\alpha \beta, \beta}^{*}(\mathbf{x}, t)+D^{(u u)}(\mathbf{x}) \ddot{u}_{\alpha}^{*}(\mathbf{x}, t)+D^{(u w)}(\mathbf{x}) \ddot{w}_{, \alpha}^{*}(\mathbf{x}, t)=0 \\
M_{\alpha \beta, \alpha \beta}^{*(w)}(\mathbf{x}, t)+D_{1}^{(w w)}(\mathbf{x}) \ddot{w}^{*}(\mathbf{x}, t)-\left[D^{(w u)}(\mathbf{x}) \ddot{u}_{\beta}^{*}(\mathbf{x}, t)+D_{2}^{(w w)}(\mathbf{x}) \ddot{w}_{, \beta}^{*}(\mathbf{x}, t)\right]_{, \beta}=-\bar{t}_{3}^{*}(\mathbf{x}, t) \\
B_{\alpha, \alpha}^{*}(\mathbf{x}, t)-B_{3}^{*}(\mathbf{x}, t)=0,
\end{gathered}
$$

Where the semi-integral fields and dimensionless coefficients $D^{(\urcorner)}(\mathbf{x})$ are defined in the Appendix. The dimensionless transversal load is defined as $\bar{t}_{3}^{*}(\mathbf{x}, t):=\left(L^{4} / D_{0} h_{0}\right) \bar{t}_{3}(\mathbf{x}, t)$, where $D_{0}=c_{11(0)}^{E}\left(h_{0}\right)^{3}$ is the bending stiffness of the homogeneous plate.

The most frequently applied boundary conditions for mechanical fields on the boundary edge of the plate are given as follows:

(i) on clamped edge:

$$
\left.u_{\alpha}^{*}(\mathbf{x}, t)\right|_{\partial \Omega}=0,\left.w^{*}(\mathbf{x}, t)\right|_{\partial \Omega}=0,\left.\frac{\partial w^{*}}{\partial \mathbf{n}}(\mathbf{x}, t)\right|_{\partial \Omega}=0
$$

(ii) on simply supported edge:

$$
\left.n_{\beta}(\mathbf{x}) T_{\alpha \beta}^{*}(\mathbf{x}, t)\right|_{\partial \Omega}=0,\left.w^{*}(\mathbf{x}, t)\right|_{\partial \Omega}=0,\left.n_{\alpha}(\mathbf{x}) n_{\beta}(\mathbf{x}) M_{\alpha \beta}^{*(w)}(\mathbf{x}, t)\right|_{\partial \Omega}=0,
$$

while for electric potential field on the boundary edge $\partial \Omega$ the following boundary conditions are admissible

$$
\begin{aligned}
& \text { (i) Dirichlet type: }\left.\phi_{0}^{*}(\mathbf{x}, t)\right|_{\partial \Omega}=\bar{\phi}_{0}(\mathbf{x}, t) \\
& \text { (ii) Neuman type: }\left.n_{\alpha}(\mathbf{x}) B_{\alpha}^{*}(\mathbf{x}, t)\right|_{\partial \Omega}=0
\end{aligned}
$$

The semi-integral fields in Eq.(4) include higher order derivatives of primary field variables, which lead to increasing inaccuracy of the solution. To overcome this problem, we decompose original $4^{\text {th }}$ order governing PDEs into coupled set of $2^{\text {nd }}$ order PDEs by introducing new field variables as

$$
m^{*}(\mathbf{x}, t):=\nabla^{2} w^{*}(\mathbf{x}, t), \quad s_{\alpha}^{*}(\mathbf{x}, t):=\nabla^{2} u_{\alpha}^{*}(\mathbf{x}, t)
$$

Then, the complet set of equations of motion for primary field variables $\left\{w^{*}(\mathbf{x}, t), m^{*}(\mathbf{x}, t), u_{\beta}^{*}(\mathbf{x}, t), s_{\beta}^{*}(\mathbf{x}, t), \phi_{0}^{*}(\mathbf{x}, t)\right\}$ is given by Eqs (4) and (7).

\section{Numerical implementation}

In this paper, we have used the strong formulation with employing the meshless approximation for primary field variables by Central Approximation Node (CAN) concept of Moving Least Square (MLS) approximation technique [10,11].

Without going into details [12], the approximation of field variable $u(\mathbf{x})$ around the central approximation node $\mathbf{x}^{q}$ can be expressed by 


$$
u(\mathbf{x}, t) \approx \sum_{a=1}^{N^{q}} \hat{u}^{\bar{a}}(t) \phi^{(q, a)}(\mathbf{x}), \quad \bar{a}=n(q, a)
$$

where $\bar{a}$ is the global number of the $a$-th node from the influence domain of $\mathbf{x}^{q}, N^{q}$ is the number of nodal points in the influence domain, and $\phi^{(q, a)}(\mathbf{x})$ is the shape function associated with the node $n(q, a)$. The CAN can be selected as the nearest node to the field point $\mathbf{X}$.

The derivatives of the field variable $u(\mathbf{x})$ can be approximated by derivatives of approximated fields (D0-approach) [4], i.e.

$$
u_{, i j k \ldots l}(\mathbf{x}, t) \approx \sum_{a=1}^{N^{q}} \hat{u}^{\bar{a}}(t) \phi_{, i j k \ldots l}^{(q, a)}(\mathbf{x}) .
$$

Since the nodal unknowns are time-dependent, the spatially discretized equations are given by the system of ODE (ordinary differential equations). For numerical solution of the system of the ODE for nodal unknowns we employ the Wilson $\theta$-method [13,14] which assumes a linear change of accelerations within the time interval $\left[t_{k}, t_{k}+\theta \Delta t\right]$, where $\Delta t$ is the time step and $t_{k+1}=t_{k}+\Delta t$.

\section{Numerical experiments}

In presented numerical investigations of thin FGPM plates under transient voltage loads within the KLT, we consider a square plate $L \times L$ with, $L=1$, and in-plane gradations of material coefficients. Poisson's ratio is assumed to be constant $v=0.3$. The material coefficients corresponding to PZT-4 [9]. The transient voltage loading is applied as $\Phi^{ \pm^{*}}(\mathbf{x}, t):=\phi^{+^{*}}\left(1-e^{-t / \tau}\right)$, where $\tau=0.0001$ and $\phi^{+^{*}}=400$. The mechanical loading is omitted, $\quad \overline{t_{3}}(\mathbf{x}, t)=0$, and the initial conditions are vanishing $u_{\alpha}(\mathbf{x}, t=0)=0$, $w(\mathbf{x}, t=0)=0, \phi_{0}^{*}(\mathbf{x}, t=0)=0$. The plate edges are traction free with clamped $(\mathrm{CE})$ boundary conditions, while the electric boundary conditions are assumed to be Dirichlet (DE) boundary condition. In the Wilson $\theta$-method, we have chosen the parameter $\theta=1.4$.

In all numerical computations, we have used a uniform distribution of nodal points (16x16 nodes) with $\delta$ being the distance between two neighbour nodes. The other parameters in the MLS-approximation have been taken as: the radius of the interpolation domain $\rho^{a}=3.001 \delta$, shape function parameter $c^{a}=\delta$, and cubic polynomial basis.

The proper correlation between the spatial and temporal discretization determines the stability of numerical solutions. The Courant-Friedrichs-Levy (CFL) criterion is usually used as the necessary but not sufficient condition for stability $|c \Delta t / \delta| \leq 1$, where $c, \Delta t$ and $\delta$ are the velocity, time step and spatial discretization step, respectively. In the case of coupled field transient problems, the proper selection of the time step becomes problematic, 
if spreading of excitations of particular fields is characterized by various velocities. Therefore, the size of the time step is variable according to the velocity of spreading of elastic and electric excitations depending on the gradation of material properties.

First of all, we shall present the time variation of electric potential (Fig.1). It can be seen that the in-plane gradation of all the material coefficients has no influence on the time variation of the electric potential field.

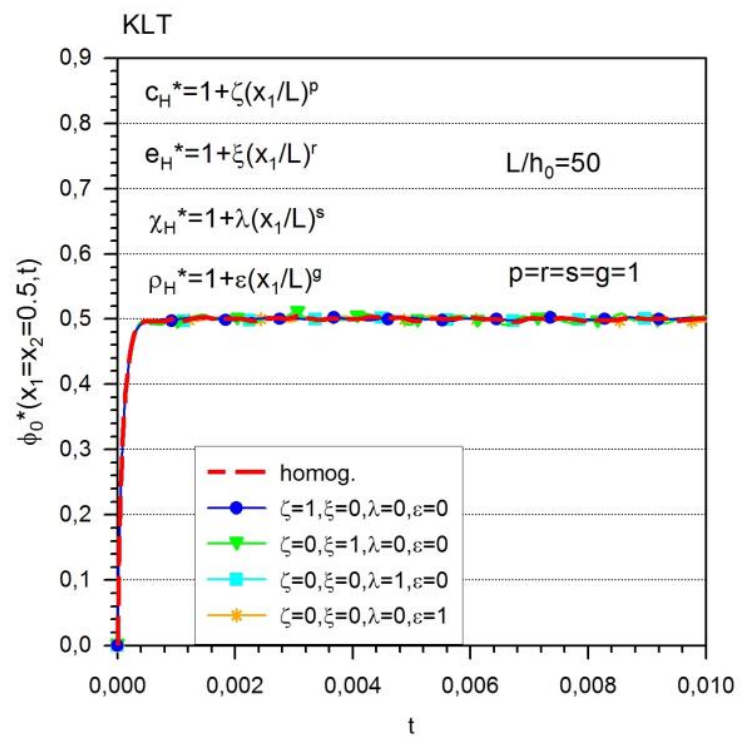

Fig. 1. Time vatiations of electric potentials of homogeneous and FGPM plates under transient voltage loading.

In what follows, we concise to presentation of numerical results for in-plane displacements of the square thin FGPM plates. The significant influence of in-plane gradation of various material coefficients on in-plane displacements can be observed on Fig. 2. The in-plane power-law gradation of elastic coefficient and the piezoelectric coefficient play the crucial role for the mechanical responses of FGPM plates subjected to voltage load, while gradation of the mass density has influence only on the frequency of oscillation of in-plane displacement. The gradation of dielectric coefficient has no influence on the time variation of in-plane displacements.

The time variations of deflections at midpoint of the FGPM plates are illustrated on Fig.3. It is shown the gradation of material coefficients has significant influence not only on the maximum amplitude of oscillation, but also on the frequency. It is observable, the inplane gradation of elastic coefficient leads to the reduction of maximum deflections, while in-plane gradation of piezoelectric coefficient has an opposite effect. In case of in-plane gradation of mass density the effect on the maximum amplitude is negligible. However, the significant influence on frequency of oscillation is observable. Also, like in the case of inplane displacements the functional gradation of dielectric coefficient has no influence on the deflection response of FGPM plate. 


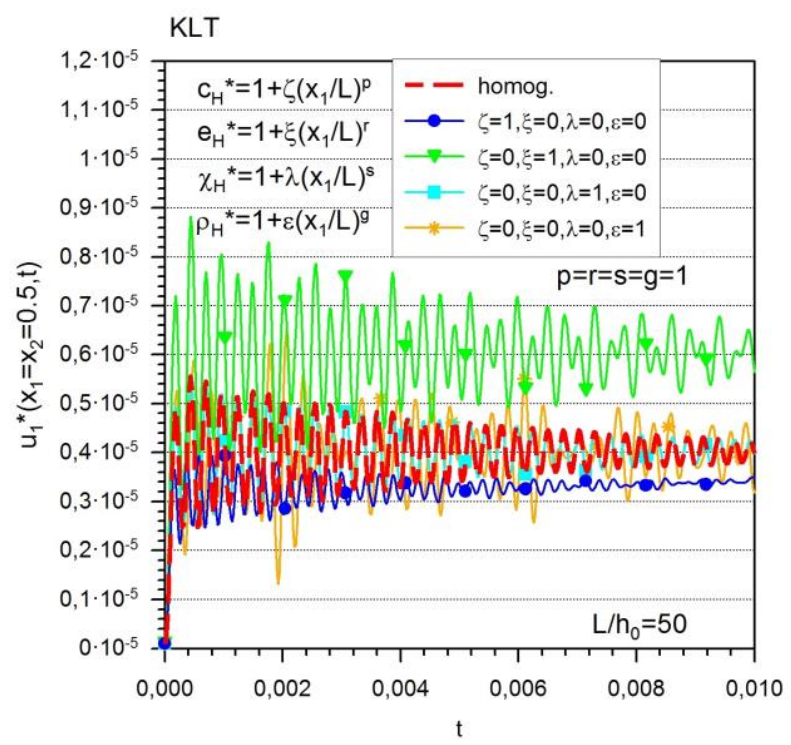

Fig. 2. Time variations of in-plane displacements of homogeneous and FGPM plates under transient voltage loading.

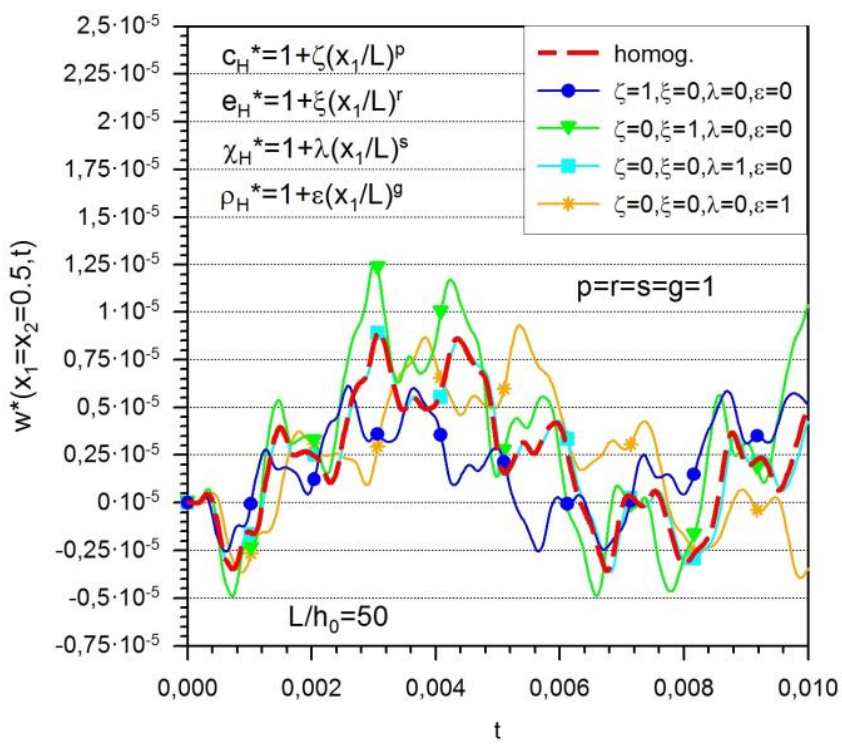

Fig. 3. Time variations of deflections of homogeneous and FGPM plates under transient voltage loading.

\section{Conclusions}

In this paper the equations of motion have been developed for FGPM plates with the assumptions of classical (KLT) plate bending theory. The in-plane functional gradation of material coefficients is considered, which makes the plate bending problems much more difficult because the equations of motion are given by PDE with variable coefficients. Combining the strong form meshless approximation, we developed an effective numerical 
method for solution of initial-boundary value problems of FGPM plates under voltage loadings. Numerical examples have been performed in order to investigate the effects of inplane gradations within FGPM plates subjected to transient voltage loading.

The financial support of the Slovak Research and Development Agency under the contract No. APVV-18-0004 is greatly acknowledged.

\section{Appendix}

The dimensionless semi-integral fields are given as

$$
\begin{aligned}
& T_{11}^{*}(\mathbf{x}, t)=\frac{L^{2}}{D_{0}} T_{11}(\mathbf{x}, t)=C_{11}^{(u u)}(\mathbf{x}) u_{1,1}^{*}(\mathbf{x}, t)+C_{12}^{(u u)}(\mathbf{x}) u_{2,2}^{*}(\mathbf{x}, t) \\
& +C_{11}^{(u w)}(\mathbf{x}) w_{, 11}^{*}(\mathbf{x}, t)+C_{12}^{(u w)}(\mathbf{x}) w_{, 22}^{*}(\mathbf{x}, t)+C_{31}^{\left(u \Phi^{-}\right)}(\mathbf{x}) \Phi^{-^{*}}(\mathbf{x}, t)+ \\
& +C_{31}^{\left(u \Phi^{+}\right)}(\mathbf{x}) \Phi^{+^{*}}(\mathbf{x}, t)+C_{31}^{\left(u \phi_{0}\right)}(\mathbf{x}) \phi_{0}^{*}(\mathbf{x}, t) \\
& T_{22}^{*}(\mathbf{x}, t)=\frac{L^{2}}{D_{0}} T_{22}(\mathbf{x}, t)=C_{12}^{(u u)}(\mathbf{x}) u_{1,1}^{*}(\mathbf{x}, t)+C_{22}^{(u u)}(\mathbf{x}) u_{2,2}^{*}(\mathbf{x}, t)+ \\
& +C_{12}^{(u w)}(\mathbf{x}) w_{, 11}^{*}(\mathbf{x}, t)+C_{22}^{(u w)}(\mathbf{x}) w_{, 22}^{*}(\mathbf{x}, t)+C_{32}^{\left(u \Phi^{-}\right)}(\mathbf{x}) \Phi^{-^{*}}(\mathbf{x}, t)+ \\
& +C_{32}^{\left(u \Phi^{+}\right)}(\mathbf{x}) \Phi^{+^{*}}(\mathbf{x}, t)+C_{32}^{\left(u \phi_{0}\right)}(\mathbf{x}) \phi_{0}^{*}(\mathbf{x}, t) \\
& T_{12}^{*}(\mathbf{x}, t)=\frac{L^{2}}{D_{0}} T_{12}(\mathbf{x}, t)=C_{66}^{(u u)}(\mathbf{x})\left(u_{1,2}^{*}(\mathbf{x}, t)+u_{2,1}^{*}(\mathbf{x}, t)\right)+2 C_{66}^{(u w)}(\mathbf{x}) w_{, 12}^{*}(\mathbf{x}, t) \\
& C_{a b}^{(u u)}(\mathbf{x})=\frac{L h_{0}}{D_{0}} \tilde{C}_{a b}^{(u u)}(\mathbf{x})=\frac{L\left(h_{0}\right)^{2}}{D_{0}} c_{a b(0)}^{E} c_{(H)}^{E}(\mathbf{x}) h^{*}(\mathbf{x}) \\
& C_{a b}^{(u w)}(\mathbf{x})=\frac{h_{0}}{D_{0}} \tilde{C}_{a b}^{(u w)}(\mathbf{x})=\frac{\left(h_{0}\right)^{3}}{D_{0}} c_{a b(0)}^{E} c_{(H)}^{E}(\mathbf{x}) h^{* 2}(\mathbf{x}) \\
& C_{3 \alpha}^{\left(u \Phi^{-}\right)}(\mathbf{x})=\frac{L^{2} \Phi_{0}}{D_{0}} \tilde{C}_{3 \alpha}^{\left(u \Phi^{-}\right)}(\mathbf{x})=\frac{L^{2} \Phi_{0}}{D_{0}} e_{3 \alpha(0)} e_{(H)}(\mathbf{x}) \\
& C_{3 \alpha}^{\left(u \Phi^{+}\right)}(\mathbf{x})=\frac{L^{2} \Phi_{0}}{D_{0}} \tilde{C}_{3 \alpha}^{\left(u \Phi^{+}\right)}(\mathbf{x})=\frac{L^{2} \Phi_{0}}{D_{0}} e_{3 \alpha(0)} e_{(H)}(\mathbf{x}) \\
& C_{3 \alpha}^{\left(u \phi_{0}\right)}(\mathbf{x})=-2 C_{3 \alpha}^{\left(u \Phi^{+}\right)}(\mathbf{x}) \\
& M_{11}^{(w)^{*}}(\mathbf{x}, t)=\frac{L^{2}}{D_{0} h_{0}} M_{11}^{(w)}(\mathbf{x}, t)=C_{11}^{(w u)}(\mathbf{x}) u_{1,1}^{*}(\mathbf{x}, t)+C_{12}^{(w u)}(\mathbf{x}) u_{2,2}^{*}(\mathbf{x}, t)+ \\
& +C_{11}^{(w w)}(\mathbf{x}) w_{, 11}^{*}(\mathbf{x}, t)+C_{12}^{(w w)}(\mathbf{x}) w_{, 22}^{*}(\mathbf{x}, t)+C_{31}^{\left(w \Phi^{-}\right)}(\mathbf{x}) \Phi^{-^{*}}(\mathbf{x}, t)+ \\
& +C_{31}^{\left(w \Phi^{+}\right)}(\mathbf{x}) \Phi^{+^{*}}(\mathbf{x}, t)+C_{31}^{\left(w \phi_{0}\right)}(\mathbf{x}) \phi_{0}^{*}(\mathbf{x}, t)
\end{aligned}
$$




$$
\begin{aligned}
& M_{22}^{(w)^{*}}(\mathbf{x}, t)=\frac{L^{2}}{D_{0} h_{0}} M_{22}^{(w)}(\mathbf{x}, t)=C_{12}^{(w u)}(\mathbf{x}) u_{1,1}^{*}(\mathbf{x}, t)+C_{22}^{(w u)}(\mathbf{x}) u_{2,2}^{*}(\mathbf{x}, t)+ \\
& +C_{12}^{(w w)}(\mathbf{x}) w_{, 11}^{*}(\mathbf{x}, t)+C_{22}^{(w w)}(\mathbf{x}) w_{, 22}^{*}(\mathbf{x}, t)+C_{32}^{\left(w \Phi^{-}\right)}(\mathbf{x}) \Phi^{-*}(\mathbf{x}, t)+ \\
& +C_{32}^{\left(w \Phi^{+}\right)}(\mathbf{x}) \Phi^{+^{*}}(\mathbf{x}, t)+C_{32}^{\left(w \phi_{0}\right)}(\mathbf{x}) \phi_{0}^{*}(\mathbf{x}, t) \\
& M_{12}^{(w)^{*}}(\mathbf{x}, t)=\frac{L^{2}}{D_{0} h_{0}} M_{12}^{(w)}(\mathbf{x}, t)=C_{66}^{(w u)}(\mathbf{x})\left(u_{1,2}^{*}(\mathbf{x}, t)+u_{2,1}^{*}(\mathbf{x}, t)\right)+2 C_{66}^{(w w)}(\mathbf{x}) w_{, 12}^{*}(\mathbf{x}, t) \\
& C_{a b}^{(w u)}(\mathbf{x})=\frac{L}{D_{0}} \tilde{C}_{a b}^{(w u)}(\mathbf{x})=\frac{L\left(h_{0}\right)^{2}}{D_{0}} c_{a b(0)}^{E} c_{(H)}^{E}(\mathbf{x}) h^{* 2}(\mathbf{x}) \\
& C_{a b}^{(w w)}(\mathbf{x})=\frac{1}{D_{0}} \tilde{C}_{a b}^{(w w)}(\mathbf{x})=-\frac{\left(h_{0}\right)^{3}}{D_{0}} c_{a b(0)}^{E} c_{(H)}^{E}(\mathbf{x}) h^{* 3}(\mathbf{x}) \\
& C_{3 \alpha}^{\left(w \Phi^{-}\right)}(\mathbf{x})=\frac{L^{2} \Phi_{0}}{D_{0} h_{0}} \tilde{C}_{3 \alpha}^{\left(\varphi \Phi^{-}\right)}(\mathbf{x})=\frac{L^{2} \Phi_{0}}{D_{0}} e_{3 \alpha(0)} e_{(H)}(\mathbf{x}) h^{*}(\mathbf{x}) \\
& C_{3 \alpha}^{\left(w \Phi^{+}\right)}(\mathbf{x})=\frac{L^{2} \Phi_{0}}{D_{0}} \tilde{C}_{3 \alpha}^{\left(\varphi \Phi^{+}\right)}(\mathbf{x})=\frac{L^{2} \Phi_{0}}{D_{0}} e_{3 \alpha(0)} e_{(H)}(\mathbf{x}) h^{*}(\mathbf{x}) \\
& C_{3 \alpha}^{\left(w \phi_{0}\right)}(\mathbf{x})=-2 C_{3 \alpha}^{\left(w \Phi^{+}\right)}(\mathbf{x}) \\
& B_{1}^{*}(\mathbf{x})=\frac{1}{\Phi_{0}} B_{1}(\mathbf{x})=C_{55}^{(D w \varphi)}(\mathbf{x}) w_{, 1}^{*}(\mathbf{x}, t)+C_{11}^{\left(D \Phi^{-}\right)}(\mathbf{x}) \Phi_{, 1}^{-^{*}}(\mathbf{x}, t)+ \\
& +C_{11}^{\left(D \Phi^{+}\right)}(\mathbf{x}) \Phi_{, 1}^{+^{*}}(\mathbf{x}, t)+C_{11}^{\left(D \phi_{0}\right)}(\mathbf{x}) \phi_{0,1}^{*}(\mathbf{x}, t) \\
& B_{2}^{*}(\mathbf{x})=\frac{1}{\Phi_{0}} B_{2}(\mathbf{x})=C_{44}^{(D w \varphi)}(\mathbf{x}) w_{, 2}^{*}(\mathbf{x}, t)+C_{22}^{\left(D \Phi^{-}\right)}(\mathbf{x}) \Phi_{, 2}^{-*}(\mathbf{x}, t)+ \\
& +C_{22}^{\left(D \Phi^{+}\right)}(\mathbf{x}) \Phi_{, 2}^{+^{*}}(\mathbf{x}, t)+C_{22}^{\left(D \phi_{0}\right)}(\mathbf{x}) \phi_{0,2}^{*}(\mathbf{x}, t) \\
& C_{a b}^{\left(D w \phi_{0}\right)}(\mathbf{x})=\frac{1}{\Phi_{0}} \tilde{C}_{a b}^{\left(D w \phi_{0}\right)}(\mathbf{x})=\frac{h_{0}}{\Phi_{0}} e_{3 \alpha(0)} e_{(H)}(\mathbf{x}) h^{*}(\mathbf{x}) \\
& C_{\alpha \beta}^{\left(D \Phi^{-}\right)}(\mathbf{x})=\frac{1}{L} \tilde{C}_{\alpha \beta}^{\left(D \Phi^{-}\right)}=-\frac{h_{0}}{L} \chi_{\alpha \beta(0)}^{\varepsilon} \chi_{(H)}^{\varepsilon}(\mathbf{x}) h^{*}(\mathbf{x}) \\
& C_{\alpha \beta}^{\left(D \Phi^{+}\right)}(\mathbf{x})=\frac{1}{L} \tilde{C}_{\alpha \beta}^{\left(D \Phi^{+}\right)}=-\frac{h_{0}}{L} \chi_{\alpha \beta(0)}^{\varepsilon} \chi_{(H)}^{\varepsilon}(\mathbf{x}) h^{*}(\mathbf{x}) \\
& C_{\alpha \beta}^{\left(D \phi_{0}\right)}(\mathbf{x})=\frac{1}{L} \tilde{C}_{\alpha \beta}^{\left(D \phi_{0}\right)}=-\frac{h_{0}}{L} \chi_{\alpha \beta(0)}^{\varepsilon} \chi_{(H)}^{\varepsilon}(\mathbf{x}) h^{*}(\mathbf{x})
\end{aligned}
$$




$$
\begin{gathered}
B_{3}^{*}(\mathbf{x}, t)=\frac{L}{\Phi_{0}} B_{3}(\mathbf{x}, t)=C_{31}^{(D u)}(\mathbf{x}) u_{1,1}^{*}(\mathbf{x}, t)+C_{32}^{(D u)}(\mathbf{x}) u_{2,2}^{*}(\mathbf{x}, t)+ \\
+C_{31}^{(D w)}(\mathbf{x}) w_{, 11}^{*}(\mathbf{x}, t)+C_{32}^{(D w)}(\mathbf{x}) w_{, 22}^{*}(\mathbf{x}, t)+C_{33}^{\left(D \Phi^{-}\right)}(\mathbf{x}) \Phi^{-*}(\mathbf{x}, t)+ \\
+C_{33}^{\left(D \Phi^{+}\right)}(\mathbf{x}) \Phi^{+^{*}}(\mathbf{x}, t)+C_{33}^{\left(D \phi_{0}\right)}(\mathbf{x}) \phi_{0}^{*}(\mathbf{x}, t) \\
C_{3 \alpha}^{(D u)}(\mathbf{x})=\frac{h_{0}}{\Phi_{0}} \tilde{C}_{\alpha \beta}^{(D u)}(\mathbf{x})=-\frac{h_{0}}{\Phi_{0}} e_{3 \alpha(0)} e_{(H)}(\mathbf{x}) \\
C_{3 \alpha}^{(D w)}(\mathbf{x})=\frac{h_{0}}{\Phi_{0} L} \tilde{C}_{\alpha \beta}^{(D w)}(\mathbf{x})=-\frac{\left(h_{0}\right)^{2}}{\Phi_{0} L} e_{3 \alpha(0)} e_{(H)}(\mathbf{x}) h^{*}(\mathbf{x}) \\
C_{33}^{\left(D \Phi^{-)}\right.}(\mathbf{x})=L \tilde{C}_{33}^{\left(D \Phi^{-)}\right.}(\mathbf{x})=\frac{8 L}{h_{0} h^{*}(\mathbf{x})} \chi_{33(0)}^{\varepsilon} \chi_{(H)}^{\varepsilon}(\mathbf{x}) \\
C_{33}^{\left(D \Phi^{+)}\right.}(\mathbf{x})=L \tilde{C}_{33}^{\left(D \Phi^{+)}\right.}(\mathbf{x})=\frac{32 L}{h_{0} h^{*}(\mathbf{x})} \chi_{33(0)}^{\varepsilon} \chi_{(H)}^{\varepsilon}(\mathbf{x}) \\
C_{3 \alpha}^{\left(D \phi_{0}\right)}(\mathbf{x})=-2 C_{3 \alpha}^{\left(D \Phi^{+}\right)}(\mathbf{x})
\end{gathered}
$$

Finally, there are given the dimensionless coefficients $D^{(\mathbb{I})}(\mathbf{x})$ used in Eq. (4)

$$
\begin{aligned}
& D^{(u u)}(\mathbf{x}):=\frac{L^{3} h_{0}}{D_{0} T^{2}} \tilde{D}^{(u u)}(\mathbf{x})=-\frac{L^{3}}{T^{2}} \frac{\rho_{0}\left(h_{0}\right)^{2}}{D_{0}} R_{1 H}(\mathbf{x}) \\
& D^{(u w)}(\mathbf{x}):=\frac{L^{2} h_{0}}{D_{0} T^{2}} \tilde{D}^{(u w)}(\mathbf{x})=-\frac{L^{2}}{T^{2}} \frac{\rho_{0}\left(h_{0}\right)^{3}}{D_{0}} R_{2 H}(\mathbf{x}) \\
& D_{1}^{(w w)}(\mathbf{x}):=\frac{L^{4}}{D_{0} T^{2}} \tilde{D}_{1}^{(w w)}(\mathbf{x})=-\frac{L^{4}}{T^{2}} \frac{\rho_{0} h_{0}}{D_{0}} R_{1 H}(\mathbf{x})=\frac{L}{h_{0}} D^{(u u)} \\
& D^{(w u)}(\mathbf{x}):=\frac{L^{3}}{D_{0} T^{2}} \tilde{D}^{(w u)}(\mathbf{x})=-\frac{L^{3}}{T^{2}} \frac{\rho_{0}\left(h_{0}\right)^{2}}{D_{0}} R_{2 H}(\mathbf{x})=\frac{L}{h_{0}} D^{(u w)}(\mathbf{x}) \\
& D_{2}^{(w w)}(\mathbf{x}):=\frac{L^{2}}{D_{0} T^{2}} \tilde{D}_{2}^{(w w)}(\mathbf{x})=-\frac{L^{2}}{T^{2}} \frac{\rho_{0}\left(h_{0}\right)^{3}}{D_{0}} R_{3 H}(\mathbf{x})
\end{aligned}
$$

in which $R_{j H}(\mathbf{x}):=\left(h^{*}(\mathbf{x})\right)^{j} \rho_{(H)}(\mathbf{x})$. 


\section{References}

1. S.S. Rao, M. Sunar, Appl. Mech. Rev. 47, 113-123 (1994).

2. X. Wang, W. Zhou, Z. Wu, X. Zhang, Comp. Str. 215, 166-177 (2019)

3. E. Carrera, S. Brischetto, P. Nali, Plates and Shells for Smart Structures (John Wiley \& Sons, Chichester, 2011).

4. N.D. Duc, P.H. Cong, V.D. Quang, Int. J. of Mech. Sci. 115-116, 711-722 (2016)

5. X.L. Chen, Z.Y. Zhao, K.M. Liew, Advances in Eng. Software 39, 121-131 (2008)

6. S. Mikaeeli, B. Behjat, Comp. Str. 154, 591-599 (2016)

7. B. Behjat, M. Salehi, A. Armin, M. Sadighi, M. Abbasi, Scientia Iranica 18, 986-994 (2011)

8. P. Lancaster, K. Salkauskas, Math Comput. 37, 141-158 (1981).

9. J. Sladek, V. Sladek, Ch. Zhang, F. Garcia-Sanche, M.Wunsche, CMC 4, 109-117 (2006)

10. V. Sladek, J. Sladek, Ch. Zhang, Comput Mech 41: 827-845 (2008)

11. V. Sladek, J. Sladek, Eng Anal Bound Elem; 34: 904-913 (2010)

12. V. Sladek,J. Sladek, L. Sator, Eng Anal Boundary Elem. 37, 348-365 (2013).

13. I. Gladwell, R. Thomas, Int J Numer Anal Methods Geomech 4: 143-158 (1980)

14. TJR. Hughes, The finite element method, (Prentice-Hall Inc, New Jersey, 1987) 